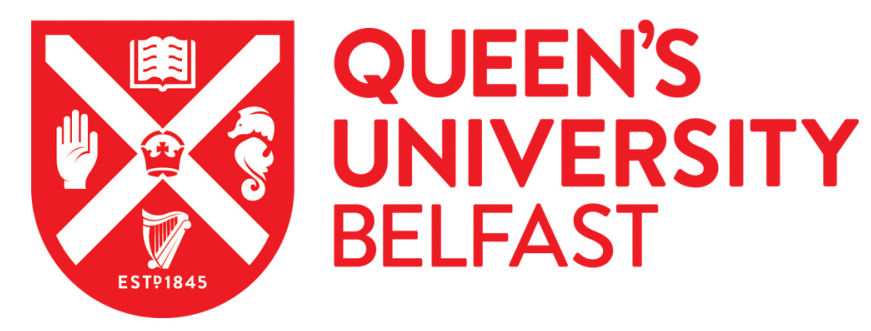

\title{
Fast Identification of Interesting Spatial Regions with Applications in Human Development Research
}

Duffy, C., P. D., Long, C., Kumar, M. S., Thorat, A., \& Dubey, A. (2018). Fast Identification of Interesting Spatial Regions with Applications in Human Development Research. In International Conference on Database and Expert Systems Applications: Proceedings (pp. 408-416). (Lecture Notes in Computer Science; Vol. 11030). Springer. https://doi.org/10.1007/978-3-319-98812-2_37

\section{Published in:}

International Conference on Database and Expert Systems Applications: Proceedings

\section{Document Version:}

Peer reviewed version

Queen's University Belfast - Research Portal:

Link to publication record in Queen's University Belfast Research Portal

\section{Publisher rights}

(c) 2018 Springer Verlag.

This work is made available online in accordance with the publisher's policies. Please refer to any applicable terms of use of the publisher.

\section{General rights}

Copyright for the publications made accessible via the Queen's University Belfast Research Portal is retained by the author(s) and / or other copyright owners and it is a condition of accessing these publications that users recognise and abide by the legal requirements associated with these rights.

Take down policy

The Research Portal is Queen's institutional repository that provides access to Queen's research output. Every effort has been made to ensure that content in the Research Portal does not infringe any person's rights, or applicable UK laws. If you discover content in the Research Portal that you believe breaches copyright or violates any law, please contact openaccess@qub.ac.uk. 


\title{
Fast Identification of Interesting Spatial Regions with Applications in Human Development Research
}

\author{
Carl Duffy ${ }^{1}$ Deepak $\mathrm{P}^{2} \quad$ Cheng Long ${ }^{2}$ \\ M. Satish Kumar ${ }^{2} \quad$ Amit Thorat $^{3} \quad$ Amaresh Dubey $^{3}$ \\ ${ }^{1}$ Sungkyunkwan University, Republic of Korea \\ ${ }^{2}$ Queen's University Belfast, UK ${ }^{3}$ Jawaharlal Nehru University, India \\ cduffy38@qub.ac.uk deepaksp@acm.org cheng.long@qub.ac.uk \\ s.kumar@qub.ac.uk \{athorat, amareshdubey\}@mail.jnu.ac.in
}

\begin{abstract}
Large-scale demographic datasets with spatial information provide a rich platform for human development research. Much emphasis is often placed on understanding deviations from dataset-level behavior across demographic attributes within spatially coherent regions, since those could point to a local condition worth addressing through regional policies, or at the other extreme, a less known success story that offers new learnings. Inspired by such scenarios, we build upon domain knowledge from HDR to devise an interestingness scoring for spatial regions and formulate the computational task of interesting spatial region identification. Accordingly, we develop a taxonomic organization of spatial regions and formulate bounds on interestingness scores, which are then leveraged to develop an efficient technique to address the task. Our search method is empirically evaluated over two real-world datasets, and is seen to record orders of magnitude of response time improvements over region enumeration. The absolute response times and the memory overheads of our approach are seen to be within highly desirable ranges, establishing the effectiveness of our solution for the task.
\end{abstract}

\section{Introduction}

Human development research (HDR) studies often target to draw insights from existence and non-existence of correlations between attributes of interest, as observed within a dataset. Recent efforts on collecting large general-purpose (secondary) datasets involving hours of interaction with each individual/household such as the Indian Human Development Survey (IHDS [2]) capture a wide variety of attributes and provide a platform for accelerating human development research through leveraging data science technologies. The IHDS dataset has been used for widely varying kinds of analysis of inter-attribute correlations; these include studying the relationship between height and cognitive achievement in children [7] and studies involving relationship between energy access and poverty [4].

In a collaborative research project bringing together researchers from both data analytics and social sciences, we explore how data analytics can enable accelerate progress on HDR. Our intent is to develop efficient methods for exploratory data analytics tailored towards zeroing in on spatial regions and inter-attribute correlations that may be, prima facie, worthy of deeper study using conventional social science methods. 


\begin{tabular}{|l|l|l|l||l|l|l|l|l|l|l|l|l|}
\hline$A F G$ & $A L M O$ & $A X Y Z$ & & $A F G$ & $A L M O$ & $A X Y Z$ & & $A F G$ & $A L M O$ & $A X Y Z$ & \\
\hline$A B C$ & $A B C$ & $A X Y Z$ & & $A B C$ & $A B C$ & $A X Y Z$ & & $A B C$ & $A B C$ & $A X Y Z$ & \\
\hline$A B C$ & $A B C D$ & $A B C D$ & $A X Y Z$ & $A B C$ & $A B C D$ & $A B C D$ & $A X Y Z$ & $A B C$ & $A B C D$ & $A B C D$ & $A X Y Z$ \\
\hline & $A$ & & $A X Y Z$ & & $A$ & & $A X Y Z$ & & $A$ & & $A X Y Z$ \\
\hline
\end{tabular}

(a)

(b)

(c)

Fig. 1: Example for Interesting Region Identification

Example: Consider a spatial dataset that is gridded into a $4 \mathrm{x} 4$ grid in Figure 1. Within each grid cell, there could be one or more attribute pairs that exhibit a different trend than what is observed across the dataset. From a computational perspective, we are interested only in the set of such attribute pairs and not on actual attributes involved in the pair; accordingly, we use a boolean feature to denote each distinct attribute pair. The boolean feature is only shown in such regions where they are 'true' (i.e., deviant behavior is observed). Thus, the top-left grid cell in the dataset in Figure 1 has recorded local behavioral deviations in three attribute pairs, denoted by $A, F$ and $G$; on the other hand, the top right cell does not contain any local (statistically significant) behavioral deviations in any attribute pairs under consideration. Figures 1 (a), (b) and (c) denote different spatial regions, indicated by shading, which could be candidates for interesting spatial regions; we limit our study to rectangular regions in this paper. The region in Figure 1(a), while being a large region, has just one attribute pair, $A$, that is present in all regions; thus, while scoring well on the region size, it fares poorly on the number of attribute-pairs on which the component cells deviate together. Figure 1(b), on the other hand, fares poorly on the size of the region, while exhibiting coherent deviations across four attribute-pairs, $A, B, C$ and $D$. Figure 1(c) is somewhat mid-way between the two earlier cases, with the region being sizeable enough, and there being three attributepairs, $A, B$ and $C$, on which the component cells record similar deviations. In particular, it presents a trade-off between region size and deviation behavior, and is intuitively more desirable than the two extremes in terms of region interestingness.

Our Contributions: In this paper, we translate the aforementioned factors that characterize a family of HDR questions into a simple formulation of interestingness for spatial regions. We then outline the computational problem of identifying the top interesting regions along with deviations that make the region interesting. We restrict our attention to rectangular regions regions in a gridded dataset, rectangular shapes being a simple and first step to ensure spatial coherence. We develop a method for efficient identification of top-k interesting regions, and illustrate that our method is able to achieve very fast response times for reasonably large datasets. The efficiency of our method as well as the effectiveness of our formulation advances the state-of-the-art in data analytics methods suitable for accelerating HDR.

\section{Related Work}

Single Type of Spatial Attributes: Research into identifying regions of interest based on a single type of boolean spatial attribute has been extensively explored both within 
the statistical community (e.g., SaTScan [5]) as well as the data mining community [8]. This has been the mainstream research direction in spatial interesting region detection. The broad framework is that there is a type of boolean attribute of interest, such as whether or not a geographically mapped person has a particular disease, and regions of interest are defined as those that are large enough while also exhibiting an unusually high or unusually low incidence of the disease. The different methods differ in terms of the kind of shapes, viz., circles, ellipses or arbitrary shapes, of regions that they are able to identify. To position this against our method, one could consider each boolean feature in our setting as a spatial attribute type; in our example in Figure 1, the boolean feature type $L$ is true only in one cell, whereas $A$ is true in nine cells in our sixteen dimensional grid. Within that analogy, our task may obviously be seen to be targeting the identification of regions based on a different form of unusualness - viz., high frequency - of a plurality of binary feature types.

Variants: While single binary spatial attribute type has largely been the target of attention, there has been work on looking at variations of the problem.[9] extends the task definition to encompass two spatial attribute types. Another work, [6], shifts the focus from discovering regions to discovering attribute pairs, and seeks to identify attribute pairs that are unusually correlated spatially across the dataset. [3] extends the discovery process from attribute-pairs to attribute-sets and is particularly targeted at continuous attributes. It intends to discover spatial regions where sets of attributes together exhibit extreme values; an example output could be $[R,\{A \uparrow, B \downarrow, C \uparrow\}]$ indicating that a spatial region described by $R$ has been found with an unusually high values of attribute $A$ and $C$ along with unusually low values of attribute $B$. Unlike [3] that focuses on approximating interestingness estimates computed over continuous attributes, we look at exact interestingness scoring over a dataset involving plurality of binary attributes. Some more variants of interesting spatial region discovery are covered in a survey [1].

\section{Task Definition \& Interestingness Scoring}

Overview: Our overall task of interest may be seen as comprising two phases: (i) identifying, for each grid cell, the set of attribute pairs that deviate from the global behavior to arrive at a spatial dataset (e.g., Figure 1 ignoring the shading) with boolean features (each denoting a distinct pair of HDR attributes), followed by (ii) identifying interesting spatial regions within that dataset along with a set of boolean features that characterize the region. We will consistently use the term features to refer to the boolean features, each of which stand for a pair of HDR-relevant demographic attributes. Since the focus of this paper is on the second phase, we now formally layout the task definition for that. Task Definition: Consider a two-dimensional grid $\mathcal{G}$ of size $p \times q$; we will use $G$ to refer to any grid cell within $\mathcal{G}$, and $G_{i j}$ to refer to the grid cell at the $i^{\text {th }}$ row and $j^{\text {th }}$ column. In enumerating rows and columns, we start from the top and left; thus, the top-left grid cell is $G_{00}$. The set of binary features that appear in (i.e., are true in) cell $G$ will be denoted as $G_{F}$. We now define a candidate spatial region as a 2-tuple, $C=\left[G_{i j}, G_{i^{\prime} j^{\prime}}\right]$, where the region $C$ encompasses all cells in the rectangular region with $G_{i j}$ as the top-left cell, and $G_{i^{\prime} j^{\prime}}$ as the bottom-right cell; $i^{\prime} \geq i$ and $j^{\prime} \geq j$ hold. We use $\operatorname{Cells}(C)$ as a shorthand to denote all cells within the region. As an example, 
the region in Figure 1(a) would be $\left[G_{00}, G_{22}\right]$. We now define $C_{F}$, the set of boolean features that characterize $C$, as an intersection of the boolean features across all cells within $C$, i.e., $C_{F}=\cap_{G \in C e l l s(C)} G_{F}$.

Our task of identifying interesting spatial regions is that of identifying the top- $k$ spatial regions that score highest on an interestingness score $\mathcal{I}(C)$, among all possible spatial regions within the grid $\mathcal{G}$.

Interestingness of a Spatial Region: We now describe the interestingness quantification for our task. As outlined in the introduction, we would like the interestingness score, $\mathcal{I}(C)$ to be directly related to both (i) $\left|C_{F}\right|$, and (ii) $|C e l l s(C)|$. Additionally, to ensure that $C_{F}$ captures most behavioral deviations within the grid cells in $C$, we would like to additionally ensure that most $G_{F}$ for cells within $C$ have a high overlap with $C_{F}$. We are now ready to outline our function:

$$
\mathcal{I}(C)=\sum_{G \in C \text { ells }(C)} \frac{\left|C_{F}\right|}{\left|G_{F}\right|}
$$

In short, our notion of interestingness computes, for every cell, $G$ in $C$, the fraction of items in $G_{F}$ that form part of each and every cell in the region (since $C_{F}$ is computed as the intersection), and adds up the cell-specific terms across all cells in $C$. The existence of a term for each cell ensures the preference for larger regions, whereas the construction of $C_{F}$ as the intersection ensures that large regions are considered only if there is enough overlap on their features. The interestingness of the rectangular regions from Figure 1 is tabulated in Table 1.

\begin{tabular}{|c|c|c|c|c|}
\hline Region & $C$ & $C_{F}$ & Score Computation & $\mathcal{I}(C)$ \\
\hline Figure 1(a) & {$\left[G_{00}, G_{22}\right]$} & $\{A\}$ & $\frac{1}{3}+\frac{1}{4}+\frac{1}{4}+\frac{1}{3}+\frac{1}{3}+\frac{1}{4}+\frac{1}{3}+\frac{1}{4}+\frac{1}{4}$ & $\mathbf{2 . 5 7}$ \\
Figure 1(b) & {$\left[G_{21}, G_{22}\right]$} & $\{A, B, C, D\}$ & $1+1$ & $\mathbf{2 . 0 0}$ \\
Figure 1(c) & {$\left[G_{10}, G_{21}\right]$} & $\{A, B, C\}$ & $1+1+1+\frac{3}{4}$ & $\mathbf{3 . 7 5}$ \\
\hline
\end{tabular}

Table 1: Interestingness of Rectangular Regions from Figure 1

\section{Our Method}

A brute force search enumerating all rectangular regions is quadratic on both the height and width of the grid, and thus is quartic on the grid edge size for square grids. In this section, we describe a search method for interesting region identification that estimates upper bounds on interestingness scores for partially seen regions, and employs early pruning of regions towards achieving much faster turnaround times for the task.

\section{Phase One: From HDR Attributes to Binary Features}

While there could be a variety of ways to identify attribute-pairs that exposit a different behavior in a grid cell as against their relationship in the entire dataset, we adopt a simple chi-square ${ }^{1}$ test to arrive at the determination. In particular, we identify, for every grid cell, the set of attribute-pairs that exhibit a statistically significant relationship.

\footnotetext{
${ }^{1}$ https://en.wikipedia.org/wiki/Chi-squared_test
} 
From among those attribute-pairs, we exclude those that have a statistically significant relationship across the entire dataset, thus arriving at the set of attribute-pairs that exhibit deviant behavior in the grid-cell when contrasted against their dataset behavior.

\section{Phase Two: Fast Identification of Interesting Spatial Regions}

Hierarchical Organization of Spatial Regions We first organize the space of all spatial regions in a structured fashion; within this structure, each region $\left[G_{i j}, G_{i^{\prime} j^{\prime}}\right]$ appears at a taxonomy of regions rooted at the single cell region corresponding to the top-left

cell, i.e., $\left[G_{i j}, G_{i j}\right]$. We allow for three kinds of 'expansions' to form the taxonomy, defined as follows:

- Downward Expansion (D): A downward expansion extends a region downward, i.e., from $\left[G_{i j}, G_{i^{\prime} j^{\prime}}\right]$ to $\left[G_{i j}, G_{\left(i^{\prime}+1\right)^{\prime} j^{\prime}}\right]$. The latter region contains additional row of $\left(j^{\prime}-j+1\right)$ cells as against the former.

- Rightward Expansion (R): $\left[G_{i j}, G_{i^{\prime} j^{\prime}}\right] \rightarrow\left[G_{i j}, G_{i^{\prime}\left(j^{\prime}+1\right)}\right]$

- Right-Down Expansion (RD): $\left[G_{i j}, G_{i^{\prime} j^{\prime}}\right] \rightarrow\left[G_{i j}, G_{\left(i^{\prime}+1\right)\left(j^{\prime}+1\right)}\right]$

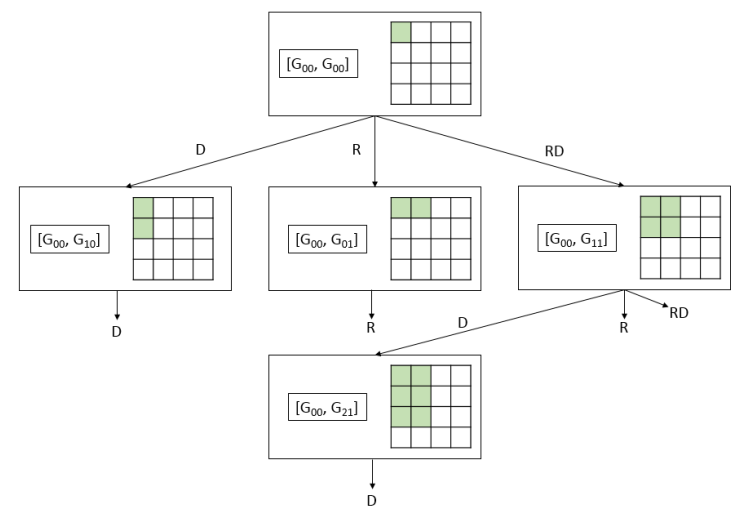

Fig. 2: Partial Taxonomy rooted at $\left[G_{00}, G_{00}\right]$ illustrating Expansion Types.

A partial view of the taxonomy rooted at $\left[G_{00}, G_{00}\right]$ appears in Figure 2, each node accompanied by an illustration of the spatial region it covers.

Restrictions on Expansions: If any sequence of $R, D$ and $R D$ expansions are allowed, a region may be encountered through multiple routes. We would like to avoid this for efficient search, and thus restrict the set of expansions. If a region is already taller than it is wide, we only allow it to be expanded into even taller regions through $D$ expansions. Similarly, for regions that are wider than tall, they can only grow wider through $R$ expansions. Square regions are allowed to grow on all three directions. Figure 2, it may be noted, depicts only the possible expansions under this set of restrictions. 
Upper Bounds of Interestingness Scores For every region, we would like to calculate the upper bound of interestingness scores that can be achieved by following each expansion type edge in the taxonomy. This needs to be computationally cheap to arrive at, while also being accurate enough to allow a search procedure relying on it to avoid exploring unpromising subtrees. One key property of our interestingness score is that a grid cell having no boolean feature being true in it is trivially uninteresting since it would push $C_{F}$ to null; our bounds computation exploits this property.

D Subtree Upper Bound: We first identify the maximum number of $D$-type expansions that can be done on the region without having to include an empty cell in the region. Now, we consider the set of all such cells within the purview of that many $D$ expansions; we will refer to this collection of cells as $\operatorname{Max} D(C)$. Each cell in $\operatorname{Max} D(C)$ can contribute a $\left|C_{F}\right| /\left|G_{F}\right|$ term to the interestingness score (Ref. Eq 1). The maximum that a cell $G \in \operatorname{Max} D(C)$ can contribute is $\frac{\min \left\{\left|C_{F}\right|,\left|G_{F}\right|\right\}}{\left|G_{F}\right|}$. This is so since the inclusion of $G$ in the region could reduce the features in the intersection to $\min \left\{\left|C_{F}\right|,\left|G_{F}\right|\right\}$ or even lower due to the other cells that are brought in due to the expansion. Thus an upper bound for regions produced by all $D$ expansions from $C$ would be:

$$
\mathcal{I}(C)+\sum_{G \in \operatorname{Max} D(C)} \frac{\min \left\{\left|C_{F}\right|,\left|G_{F}\right|\right\}}{\left|G_{F}\right|}
$$

However, this computation can be expensive when $\operatorname{Max} D(C)$ is large due to each cell having to be considered separately. Thus, we further coarsen the upper bound as:

$$
U B_{D}(C)=\mathcal{I}(C)+|\operatorname{Max} D(C)| \times \frac{\min \left\{\left|C_{F}\right|, \min \left\{\left|G_{F}^{\prime}\right| \mid G^{\prime} \in \operatorname{Max} D(C)\right\}\right\}}{\min \left\{\left|G_{F}^{\prime}\right| \mid G^{\prime} \in \operatorname{Max} D(C)\right\}}
$$

It is easy to verify that $U B_{D}(C)$ is an upper bound of Eq.2; we omit a formal proof for space constraints.

R Subtree Upper Bound: This bound is achieved by replacing $\operatorname{Max} D(C)$ above by $\operatorname{Max} R(C)$.

RD Subtree Upper Bound: Unlike $R$ and $D$ subtrees that are chains of $R$ and $D$ expansions respectively, the $R D$ subtree would involve regions that can be reached by a sequence of $R D$ expansions followed by $R$ or $D$ chains in addition to those that can be reached purely by $R D$ expansions. Accordingly, $\operatorname{Max} R D(C)$ would be defined as:

$$
\begin{aligned}
& \operatorname{Max} R D(C)=(\operatorname{ExpRD}(C, \operatorname{maxr} d(C))-C) \cup \\
& \underset{1 \leq i \leq \operatorname{maxrd}(c)}{\cup}(\operatorname{Max} D(\operatorname{ExpRD}(C, i) \cup \operatorname{MaxR}(\operatorname{ExpRD}(C, i))
\end{aligned}
$$

where $\max R D(C)$ is the number of $R D$ expansions possible without having to include an empty cell, and $\operatorname{Exp} R D(C, n)$ indicates the spatial region obtained by $n$ consecutive $R D$ expansions from $C$. The $R D$ upper bound then has a similar construction as for $D$, with $\operatorname{Max} R D(C)$ taking the place of $\operatorname{Max} D(C)$. 


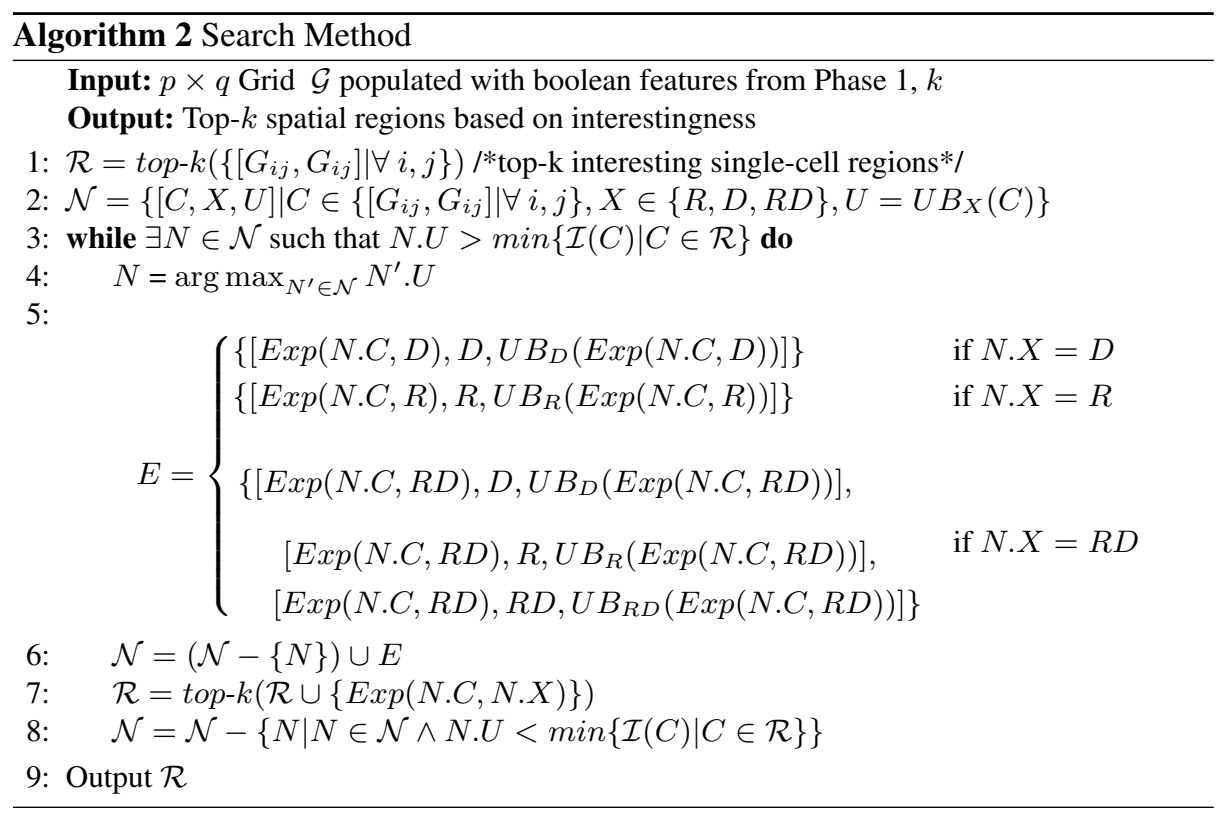

The Search Method Having defined the construction of the taxonomy of spatial regions and upper bound computation for sub-trees in the taxonomy, we now outline a search method in Algorithm 2 for identifying top- $k$ interesting spatial regions. The search process follows a best-first expansion approach exploring the space of spatial regions guided by the upper bounds. We use top- $k(\ldots)$ as a function that retains only the top-k regions in the supplied set based on their interestingness scores. The candidate set is progressively expanded through the search process, and the result set $\mathcal{R}$ is kept updated across the equations, and output at the end as the result set.

\section{Experimental Evaluation}

Datasets Our task was motivated by the need for efficient bootstrapping of HDR over the IHDS dataset [2]. This dataset captures the data from over 200,000 individuals across hundreds of attributes in India, gridded into a $130 \times 135$ grid. Since the focus of our paper is on Phase 2, which could operate upon any spatial dataset, we collected a dataset of UK POI types from a popular community initiative ${ }^{2}$, to form a second dataset for evaluation. This dataset contained $181 \mathrm{k}$ points of interest along with their types, which were placed on a $110 \times 130$ grid $(14300$ cells $)$ using just their types.

Evaluation Measures The improvement in response time against region enumeration is the primary measure of evaluation. Our method needs to maintain a set of candidates

\footnotetext{
${ }^{2}$ https://www.pocketgpsworld.com/modules.php?name=POIs
} 
(i.e., $\mathcal{N}$ ) incurring a non-constant memory overhead, making that a factor of consideration. Thus, the maximum size of $\mathcal{N}$ across all iterations, denoted as $\max N$, is the memory requirement of our search method, which forms a second evaluation measure.

Evaluation on Response Times Our method recorded significant improvements on response times on both datasets, with many orders of magnitude faster responses achieved on IHDS and upto 70 times faster responses on the POI dataset. In particular, the response times on the IHDS dataset are seen to be of the order of hundreds of milliseconds, and sub-second response times are achieved on the POI dataset, indicating the effectiveness of our pruning and search method.

Memory Overhead MaxN was recorded as 53610 for IHDS and 56123 for POI. Using a generous estimate of 25 bytes to store each candidate, this corresponds to a memory overhead of just over $1 \mathrm{MB}$, a very modest requirement for modern machines.

\section{Conclusions}

We considered the problem of interesting spatial region identification over a dataset with a plurality of boolean attribute types, inspired by the task of bootstrapping human development research from a demographic dataset. We outlined a notion of interestingness and developed an efficient method for identifying top- $k$ interesting rectangular spatial regions. Through empirical evaluation, we illustrated that our method achieves vast improvements over region enumeration with very modest memory overheads.

\section{References}

1. Deepak, P.: Anomaly detection for data with spatial attributes. In: Unsupervised Learning Algorithms, pp. 1-32. Springer (2016)

2. Desai, S., Vanneman, R.: National council of applied economic research, new delhi. india human development survey (ihds), 2005. icpsr22626-v11. Ann Arbor, MI: Inter-university Consortium for Political and Social Research [distributor] pp. 02-16 (2016)

3. Eick, C.F., Parmar, R., Ding, W., Stepinski, T.F., Nicot, J.P.: Finding regional co-location patterns for sets of continuous variables in spatial datasets. In: SIGSPATIAL. p. 30. ACM (2008)

4. Khandker, S.R., Barnes, D.F., Samad, H.A.: Are the energy poor also income poor? evidence from india. Energy policy 47, 1-12 (2012)

5. Kulldorff, M.: A spatial scan statistic. Communications in Statistics-Theory and methods 26(6), 1481-1496 (1997)

6. Shekhar, S., Huang, Y.: Discovering spatial co-location patterns: A summary of results. In: International symposium on spatial and temporal databases. pp. 236-256. Springer (2001)

7. Spears, D.: Height and cognitive achievement among indian children. Economics \& Human Biology 10(2), 210-219 (2012)

8. Telang, A., Deepak, P., Joshi, S., Deshpande, P., Rajendran, R.: Detecting localized homogeneous anomalies over spatio-temporal data. Data Mining and Knowledge Discovery 28(5-6), 1480-1502 (2014)

9. Wang, S., Huang, Y., Wang, X.S.: Regional co-locations of arbitrary shapes. In: International Symposium on Spatial and Temporal Databases. pp. 19-37. Springer (2013) 